\title{
Etude de la régénération naturelle contrôlée en forêt tropicale humide de Guadeloupe. \\ II - Installation et croissance des semis après les coupes d'ensemencement
}

\author{
M. DUCREY (*) et P. LABBE \\ INRA, Station de Recherches forestières \\ Centre de Recherches des Antilles et de la Guyane \\ Domaine Duclos, F 97170 Petit-Bourg, Guadeloupe
}

\section{Résumé}

Une étude de la régénération naturelle provoquée et assistée a ćté entreprise en forêt tropicale humide de Guadeloupe à partir de décembre 1979.

Le dispositif expérimental est constitué de 5 parcelles dont deux ont subi une coupe d'ensemencement dite forte ayant enlevé $28 \mathrm{p} .100$ du nombre de tiges et $20 \mathrm{p} .100$ de la surface terrière, deux autres une coupe d'ensemencement dite faible ayant enlevé 12 p. 100 du nombre de tiges et 5 p. 100 de la surface terrière, la cinquième étant le témoin sous forêt naturelle. Cet cnsemble de 5 parcelles a été répété en 4 "forêts» différentes réparties du Nord au Sud de la Guadeloupe.

Le bilan actue! est présenté à partir des données recueillies pendant les 3 années qui ont suivi les coupes d'ensemencement ct concerne l'effet des paramètres expérimentaux : la forêt, l'espèce (Dacryodes excelsa, Amanoa caribaea, Simaruba amara et Richeria grandis) et le type de coupe d'ensemencement sur la densité des semis, leur évolution au cours du temps, le pouvoir de régénération de chaque espèce (nombre de semis par semencier potentiel) et la croissance des semis.

Le nombre de semis est significativement différent d'une forêt à l'autre (entre 21000 et 64000 par hectare) et d'une espèce à l'autre $(14000$ par hectare pour Dacryodes excelsa, 22000 pour Amanoa caribaea, 1800 pour Simaruba amara et 1250 pour Richeria grandis. Il n'y a pas d'effet du type de coupe sur la densité des semis en raison du nombre aifférent de semenciers d'une parcelle à l'autre. Par contre, le pouvoir de régénération pour l'ensembie des espèces est significativement plus élevé sous coupes fortes et faibles que sous le témoin.

La croissance en hauteur des semis a été étudiée pendant environ 1 annéc dans 2 des 4 forêts. On observe des différences significatives d'une forêt à l'autre et aussi entre espèces. L'accroissement total annuel a été de $15,1 \mathrm{~cm}$ pour Simaruba amara, $10.9 \mathrm{~cm}$ pour Amanoa caribaea et $4,8 \mathrm{~cm}$ pour Dacryodes excelsa. Les coupes d'ensemencement ont un effet positif sur l'accroissement en hauteur qui est pour l'ensemble des espèces de $17,7 \mathrm{~cm}$ pour la coupe forte, $6,3 \mathrm{~cm}$ pour la coupe faible et $3,3 \mathrm{~cm}$ pour le témoin. L'accroissement en hauteur pour chaque espèce est lié positivement au rayonnement solaire mesuré sous le couvert et aussi à l'inverse de la surface terrière.

(*) Adresse actuelle : INRA, Station de Sylviculture méditerranćenne, avenue A.-Vivaldi, F 84000 Avignon. 
Ces résultats donnent une première idée du tempérament des espèces étudiées que l'on peut classer de la plus sciaphile à la plus héliophile dans l'ordre suivant : Dacryodes excelsa, Amanoa caribaea, Richeria grandis et Simaruba amara. Cette classification permet de comprendre la place respective de chaque espèce dans la forêt naturelle et de tirer les premières conclusions sylvicoles de cette expérimentation.

\section{Introduction}

Nous avons entrepris l'étude de la régénération naturelle contrôlée et assistée de la forêt tropicale humide de Guadeloupe dans un double but. Il s'agissait tout d'abord de répondre à la demande de l'Office National des Forêts qui souhaite, dans le cadre de l'Aménagement de la forêt soumise de Guadeloupe, traiter les parties les plus riches de cette forêt en futaie irrégulière d'essences locales (O.N.F., 1979). Il s'agissait aussi, au plan scientifique, d'acquérir les connaissances les plus complètes possibles sur l'écologie des principales espèces précieuses afin de mieux comprendre ce qui se passe lors des différentes opérations sylvicoles qui interviennent pendant la phase de régénération naturelle. Ces connaissances écologiques nouvelles nous permettront de proposer, si le besoin s'en fait sentir, des sylvicultures différentes telles que, par exemple, la plantation en plein de ces espèces locales.

Dans un article précédent (Ducrey \& LabBÉ, 1985) nous avons présenté en détail les motivations de cette étude en partant d'une part d'une analyse bibliographique des différentes études de la régénération naturelle des forêts tropicales, essentiellement des forêts denses humides, et d'autre part de la composition floristique de la forêt hygrophile de Guadeloupe. A cette occasion nous avons donné les principales caractéristiques (anatomie, morphologie, bois) des essences commercialement intéressantes : le gommier (Dacryodes excelsa Vahl,, Burseraceae), le bois rouge carapate (Amanoa caribaea Kr. et Urb., Euphorbiaceae), le marbri (Richeria grandis Vahl., Euphorbiaceae), l'acajou blanc (Simaruba amara Aubl., Simarubaceae), le palétuvier jaune (Symphonia globulifera L., Clusiaceae), le mauricif patagon (Byrsonima coriacea, Malpighiaceae), le résolu (Chimarrhis cymosa Jacq., Rubiaceae) et le bois doux chypre (Phoebe elongata Vahl, lauraceae).

Nous avons ensuite décrit la méthode de régénération naturelle que nous expérimentons ainsi que le dispositif mis en place pour étudier les différentes phases de cette régénération. Nous avons mis l'accent sur la caractérisation de l'état initial des parcelles expérimentales ainsi que sur la quantification des coupes d'ensemencement fortes et faibles qui constituent la première phase active de la régénération naturelle.

Dans le présent article, nous nous proposons de faire un premier bilan de cette expérimentation. Ce bilan, effectué à partir des données recueillies au plus tard pendant le premier semestre de 1983, soit 18 à 40 mois après les coupes d'ensemencement, permet de caractériser l'effet des coupes d'ensemencement sur la densité et la croissance des semis d'espèces précieuses avant que n'interviennent les premières coupes secondaires.

\section{Le dispositif expérimental}

L'ensemble des dispositifs expérimentaux a été décrit en détail dans l'article précédent (DUCREY \& LABBÉ, 1985). Nous n'en rappellerons que ce qui est strictement nécessaire à la compréhension de cet article. 


\subsection{Les dispositifs sylvicoles}

Quatre dispositifs ont été installés entre 1979 et 1981 dans 4 forêts de la zone hygrophile réparties du Nord au Sud de la Côte-Au-Vent de la Guadeloupe : Choisy, Jules, Sarcelle et la Digue. Chaque dispositif a une surface expérimentale de 9 hectares et une surface totale de 16 hectares. Il contient 2 parcelles de 2 hectares dans lesquelles nous avons fait une coupe d'ensemencement dite forte qui a enlevé en moyenne 28 p. 100 du nombre de tiges correspondant à 20 p. 100 de la surface terrière, 2 parcelles de 2 hectares dans lesquelles nous avons fait une coupe d'ensemencement dite faible qui a enlevé en moyenne 12 p. 100 du nombre de tiges correspondant à 5 p. 100 de la surface terrière et une parcelle d'un hectare de forêt naturelle servant de témoin.

\subsection{Les observations microclimatologiques}

Dans le dispositif installé en forêt de Sarcelle nous avons effectué des observations microclimatologiques pour caractériser les modifications créées par les coupes d'ensemencement. La température de l'air sous abri (maximum et minimum) ainsi que le pouvoir évaporant de l'air (évaporomètre piche) ont été mesurés hebdomadairement da:as chacune des 5 parcelles expérimentales pendant une période de 7 mois d'octobre 1981 à mai 1982.

L'estimation du microclimat lumineux a été faite en utilisant la méthode des photographies hémisphériques (DuCREY, 1975). Des photographies hémisphériques ont été prises à l'emplacement des piquets délimitant les 25 placettes de chaque parcelle, c'est-à-dire à raison de 36 clichés par parcelle. On n'a retenu par la suite que la valeur moyenne caractérisant chaque parcelle expérimentale.

\subsection{L'observation des semis}

Pour dénombrer les semis nous avons réalisé un inventaire statistique en utilisant un taux d'échantillonnage de 1 p. 100. Ce taux a été choisi d'après les conclusions de BARNARD (1950) qui préconise dans le cadre de l'échantillonnage linéaire par bandes, fréquemment utilisé en forêt tropicale, un taux d'échantillonnage variant de 0,5 à 2 p. 100 selon la taille des semis inventoriés. Pour ce qui nous concerne nous avons choisi un échantillonnage systématique avec 100 placeaux circulaires de $2 \mathrm{~m}^{2}$ par parcelle de 2 ha. Ces placeaux ont été disposés à raison de 4 par placette et sont situés sur les diagonales à 5 mètres des angles de chaque placette. Cette disposition a été choisie pour faciliter le repérage des placeaux.

Les mesures de croissance ont été effectuées dans chaque parcelle au niveau des 64 placeaux intérieurs. (On a dû éliminer les placeaux périphériques, les travaux sylvicoles n'ayant pas encore été faits dans les zones tampon au commencement des mesures.) A proximité de chaque placeau, nous avons choisi un bois rouge, un gommier et un acajou blanc. Quelquefois la présence plus fréquente d'une autre espèce nous a amenés à prendre en plus un marbri ou un palétuvier jaune. Les semis ont été choisis dans les diverses classes de hauteur présentes, c'est-à-dire entre 10 et 100 centimètres.

Les dénombrements de semis ainsi que les mesures de croissance ont été faits à intervalles très irréguliers (les plus proches étant séparés de 2 mois) en fonction 
des possibilités et couvrent dans le cadre de cet article la période allant d'avril 1981 à juillet 1983.

\subsection{Analyse statistique des données}

Les données recucillies au niveau des placeaux, en particulier le dénombrement des semis, ne suivent pas toujours une distribution normale (voir chapitre 3.2.). D'autre part certaines données ont été synthétisées directement au niveau des parcelles. Pour ces 2 raisons nous avons effectué l'analyse des données à partir des moyennes obtenues sur chaque parcelle.

Le modèle d'analyse de variance retenu est le suivant :

$$
Y_{i j k l}=M+A_{i}+B_{j}+C_{k}+(A B)_{i j}+(A C)_{j k}+(B C)_{j k}+E_{i j k l}
$$

11 permet d'étudier l'effet des facteurs

$$
\begin{aligned}
& \text { A : FORET } \\
& \text { B : ESPECE } \\
& \text { C : COUPE }
\end{aligned}
$$

et des intéractions d'ordre 1 entre ces trois facteurs sur les différentes variables.

Les moyennes de chaque variable pour les différents niveaux des facteurs ont été comparées entre elles grâce à un test de Duncan.

\section{Installation des semis d'essences précieuses}

La première opération sylvicole effectuée lors de l'installation des dispositifs expérimentaux, donc avant les coupes d'ensemencement a été un nettoiement général des parcelles, destinć à faciliter leur pénétration. On a ainsi enlevé toutes les herbes, broussailles et lianes ainsi que tous les brins d'espèces indésirables d'une hauteur inférieure à 4 mètres. Les brins d'espèces précieuses ont en principe été préservés mais les jeunes semis en particulier ont souffert du piétinement.

Il n'a pas élé possible d'effectuer des comptages de semis avant le nettoiement des parcelles ni, sauf à la Digue, immédiatement après les coupes d'ensemencement. Ceci ne permet donc pas d'avoir véritablement le point de départ de la régénération. Aussi, nous avons concentré notre analyse sur les semis, préexistants ou non, présents à un moment donné et leur évolution au cours du temps.

Il n'existe pas beaucoup d'indications sur la floraison et la fructification des cspèces qui nous intéressent. Seul Marshal. (1939) donne quelques renseignements concernant les îles de Trinidad et Tobago, renseignements qu'il faudrait nuancer car ces îles sont plus au Sud que la Guadeloupe et la succession des saisons n'y est pas rigoureusement la même qu'en Guadeloupe. Le déterminisme de la floraison semble peu lié au photopériodisme dont les variations sont d'ailleurs très faibles mais davantage rattaché aux saisons hydriques, elles-mêmes très variables d'une année à l'autre : il peut ainsi y avoir plusieurs floraisons et fructifications au cours d'une même année. De plus chaque espèce se situe différemment par rapport aux saisons hydriques 
sèches et humides. Ceci explique que dans un premier temps nous n'ayons pas tenu compte des périodes de fructification pour établir le calendrier des interventions sylvicoles.

\subsection{Dénombrement des semis au cours du 4" trimestre 1982}

\subsection{Densité des semis}

L'ensemble des résultats est reporté dans le tableau 1. On note tout d'abord le nombre élevé de semis qui atteint, toutes espèces précicuses confondues (et pour une hauteur de $10 \mathrm{~cm}$ à 1 mètre done incluant les préexistants éventuels), 40000 semis par hectare pour Jules, 64000 pour Sarcelle, 34000 pour la Digue et 21000 pour Choisy.

Le nombre de semis de gommier est toujours assez faible et compris entre 1 et 4 par placeau de $2 \mathrm{~m}^{2}$ soit entre 5000 et 20000 par hectare. On observe des différences de densité d'une parcelle à l'autre et en particulier, la densité dans les parcelles témoins de la Digue et de Sarcelle est plus faible que dans les parcelles éclaircies. Mais on ne peut pas en tirer d'enseignement général quant à l'effet des coupes, notamment en raison du nombre différent, d'une parcelle à l'autre, des semenciers potentiels.

Les semis de bois rouge, par comparaison avec le gommier, sont relativement abondants au moins pendant la phase de régénération que nous étudions et on trouve fréquemment de véritables brosses de semis : jusqu'à 100 par mètre carré. Le nombre moyen de semis par parcelle varie de 0 (pas de bois rouge dans la parcelle 3 de Choisy) à près de 60000 par hectare dans la parcelle 2 de Sarcelle ce qui dénote une grande variabilité d'une parcelle à l'autre et d'un dispositif à l'autre. Par contre on ne peut pas mettre en évidence de différences entre les 2 types de coupe d'ensemencement ni entre les coupes et le témoin.

L'acajou blanc est lui aussi présent dans tous les dispositifs en nombre souvent très faible : de 350 par hectare à Jules à 3750 par hectare à Sarcelle. Son port particulier : feuilles composées rattachées en rosette près du bourgeon terminal et avec 13 à 15 paires de folioles le rend facilement reconnaissable. C'est pourquoi, par simple observation, on a tendance à en surestimer la densité dans les régénérations.

Les semis de marbri sont eux aussi présents dans tous les dispositifs, en nombre moins grand que l'acajou blanc, et à l'exception de Choisy où l'on en rencontre très peu mais où il y a aussi très peu de semenciers.

Les palétuviers jaunes sont plus rares, aussi ne les rencontre-t-on pas, et a fortiori leurs semis, dans tous les dispositifs. C'est le cas en particulier de Sarcelle où il n'y a aucun palétuvier jaune et de Choisy et Jules où on n'en trouve que dans certaines parcelles.

\subsection{Relations entre semis et semenciers potentiels}

Nous avons vu dans l'article précédent (DuCREY \& LABBÉ, 1985) que la composition en espèces précieuses était très différente d'une parcelle à l'autre et d'un dispositif à l'autre. Ceci nous amène à comparer le nombre de semis d'une espèce au nombre d'arbres de ceite même espèce. Nous considérons ainsi que tous les 


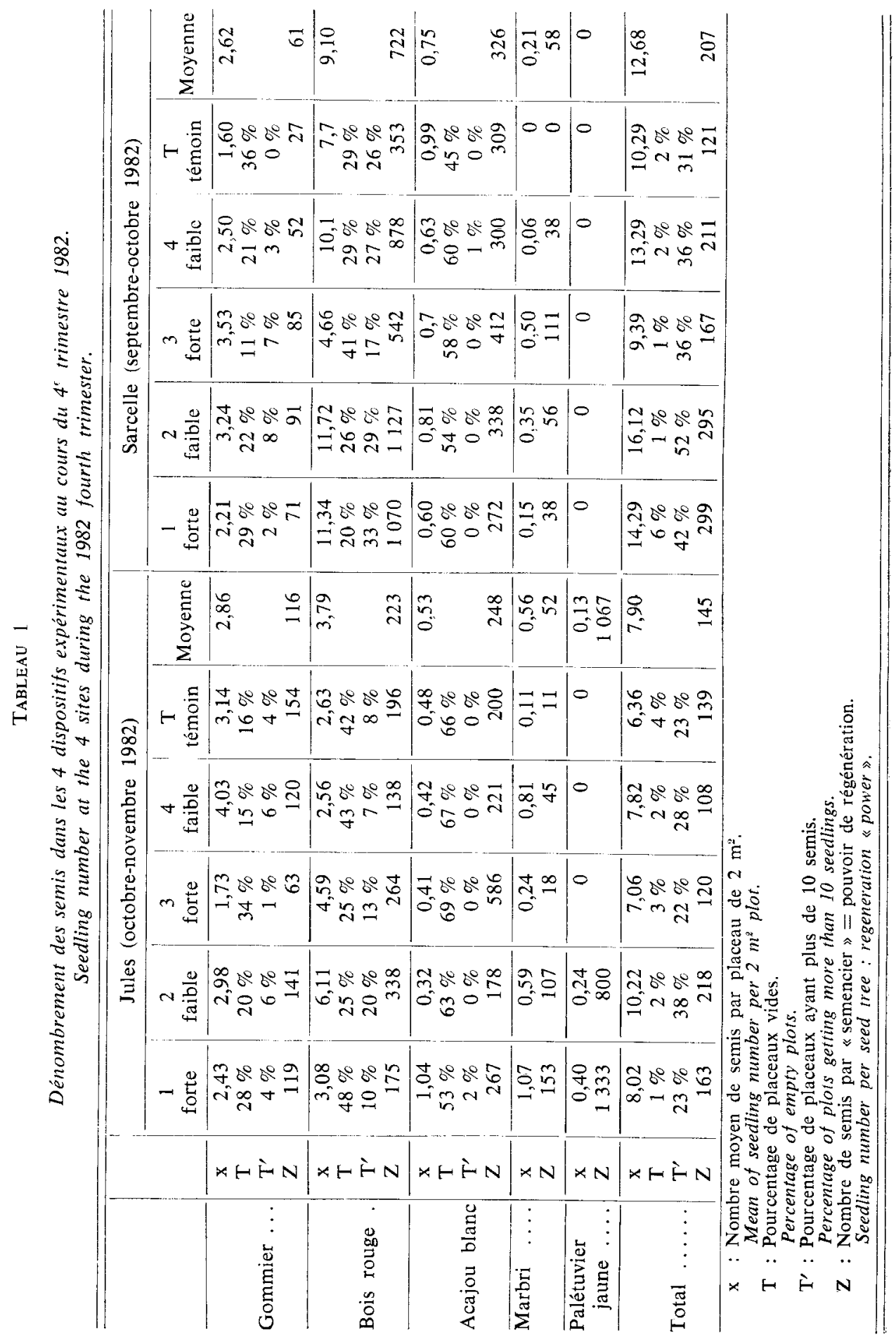




\begin{tabular}{|c|c|c|c|c|c|c|c|}
\hline \multirow{6}{*}{ 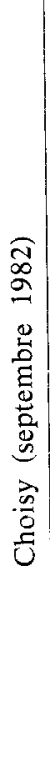 } & $\begin{array}{l}0 \\
\text { E } \\
\text { D } \\
\text { है } \\
\Sigma\end{array}$ & $\stackrel{8}{\circ} \quad 8$ & $\stackrel{\text { q }}{-}$ & $\overline{0}$ & $\delta_{0}^{0}$ & 표용 & $\stackrel{\substack{\infty \\
\forall}}{\forall}$ \\
\hline & 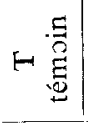 & 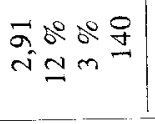 & 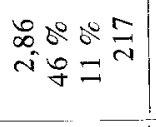 & $\ddot{0}$ & 00 & 0 & $\stackrel{\infty}{i n}$ \\
\hline & $+\underset{\pi}{\stackrel{0}{0}}$ & 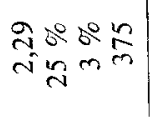 & 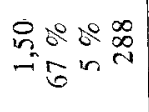 & 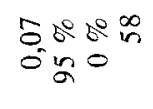 & 00 & 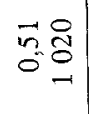 & 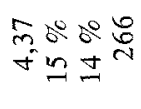 \\
\hline & $m \stackrel{\frac{0}{0}}{\frac{\pi}{\pi}}$ & 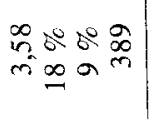 & $\begin{array}{cc}08 & 0 \\
8 & \vdots \\
0\end{array}$ & 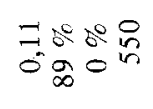 & $\overrightarrow{0}=$ & 0 & 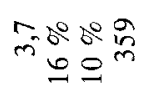 \\
\hline & $\sim \underset{\square}{5}$ & 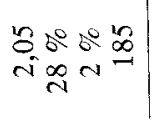 & 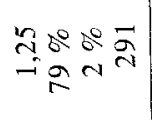 & $\begin{array}{l}\infty \\
=00 \\
0 \\
0\end{array}$ & 00 & 0 & 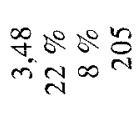 \\
\hline & $\neg \stackrel{\oplus}{\stackrel{ \pm}{0}}$ & 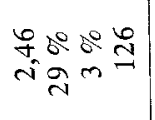 & $\begin{array}{l}78005 \\
-60\end{array}$ & $\begin{array}{l}I x: \bar{c} \\
\ln _{\infty} 0\end{array}$ & $5_{0}^{0}$ & ఫ̊용 & 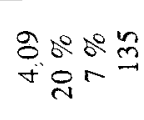 \\
\hline \multirow{8}{*}{ 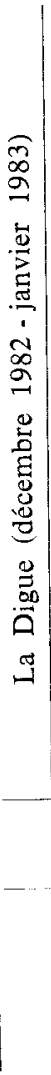 } & $\begin{array}{l}0 \\
\Xi \\
0 \\
2 \\
0 \\
\Sigma\end{array}$ & $\stackrel{2}{i} \quad \stackrel{0}{-1}$ & $\underset{m}{\stackrel{N}{F}} \quad \stackrel{N}{J}$ & 5 & $\overbrace{0}^{n}$ & $\begin{array}{ll}0 & n \\
2 & \infty \\
0 & m \\
- & -1\end{array}$ & $\begin{array}{l}\vec{\infty} \\
\hat{0}\end{array}$ \\
\hline & 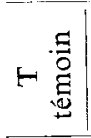 & $\begin{array}{lll}-\tilde{6} & 20 & 0 \\
-1 & 0\end{array}$ & $\begin{array}{l}m \& 206 \\
= \pm m\end{array}$ & 8 & $\hat{O}_{0} m$ & $\begin{array}{l}1 \\
0 \\
0\end{array}$ & $\vec{n}$ \\
\hline & $+\underset{\stackrel{0}{0}}{0}$ & 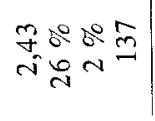 & 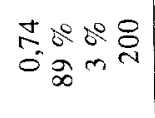 & $\stackrel{0}{0}$ & $m_{0}^{m}$ & $\frac{n}{n} \frac{n}{m}$ & $\exists$ \\
\hline & $\mathrm{m} \underset{0}{\stackrel{0}{\pi}}$ & 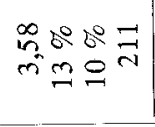 & 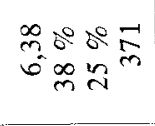 & $\begin{array}{l}\text { J } \\
0\end{array}$ & $\frac{m}{0} \bar{n}$ & $=\frac{n}{2}$ & 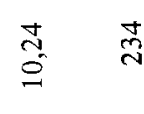 \\
\hline & $\sim \stackrel{\frac{2}{0}}{\stackrel{5}{7}}$ & 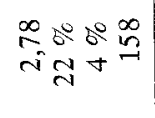 & 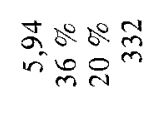 & $\stackrel{8}{0}$ & స్ & $\begin{array}{l}\text { 용 } \\
0 \\
0\end{array}$ & $\begin{array}{l}8 \\
0\end{array}$ \\
\hline & \multirow[t]{3}{*}{$-\underset{\stackrel{D}{0}}{0}$} & 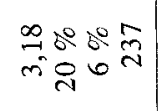 & 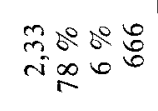 & 8 & $\begin{array}{l}\operatorname{m}_{n}^{2} \\
0^{n}\end{array}$ & $\frac{n}{0}=$ & $\frac{ \pm}{6}$ \\
\hline & & $x \in \grave{H} N$ & $x \mapsto \dot{H} N$ & $x \in \grave{H}$ & $4 N$ & $\times N$ & $x \in E$ \\
\hline & & 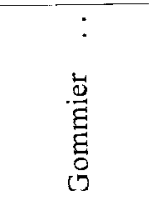 & $\begin{array}{l}0 \\
0 \\
0 \\
0 \\
0 \\
0 \\
0 \\
0\end{array}$ & 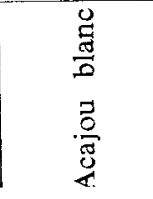 & 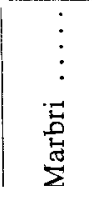 & 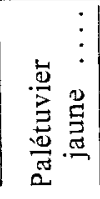 & · \\
\hline
\end{tabular}




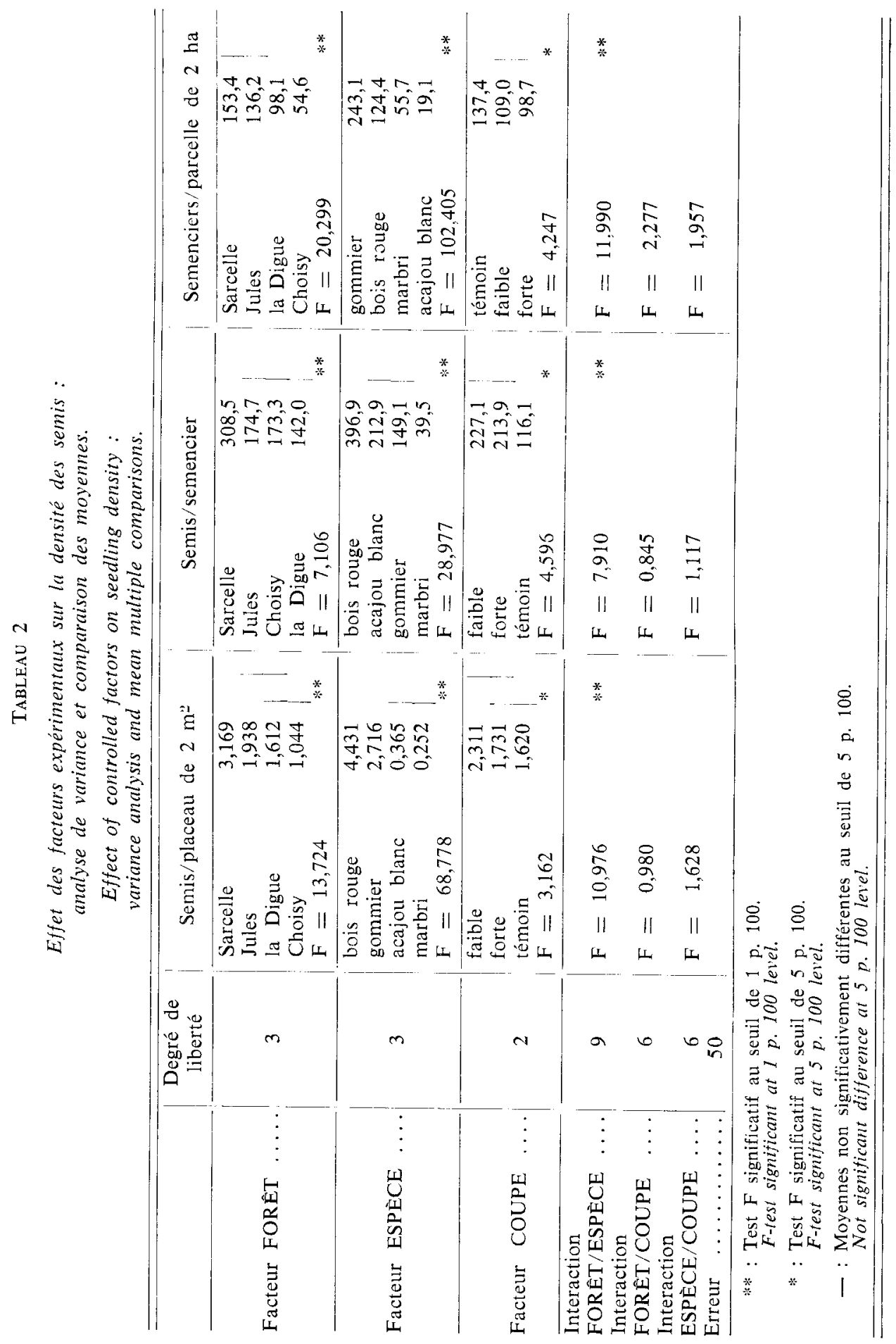


arbres pris en compte dans l'inventaire (diamètre supérieur à $12,5 \mathrm{~cm}$ ) sont des scmenciers potentiels. Cette affirmation est bien entendu à nuancer, car on ne sait pas à partir de quel âge ni de quelle taille les différentes espèces sonî fructifères. On suppose aussi que tous les arbres portent des fruits ce qui n'est pas le cas des espèces dioïques, mais on n'a pas de renseignements suffisamment précis sur la biologie florale des espèces étudiées pour pouvoir en être certain. Nous avons donc défini pour chaque parcelle le «pouvoir de régénération » de chaque espèce comme étant le rapport du nombre de semis au nombre de senenciers potentiels. Ces résultats figurent aussi sur le tableaı 1.

Pour le gommier on constate que des densités de semis identiques d'un dispositif à l'autre ont été obtenues avec des nombres de semenciers potentiels très différents et que le «pouvoir de régénération» varie de 61 à 200 semis par semencier de Sarcelle à Choisy.

Pour le bois rouge, si on se réfère aux valeurs moyennes de Jules et de la Digue en ce qui concerne le nombre de semenciers et le nombre de semis par semenciei on voit que Choisy a relativement peu de semis en raison du nombre peu élevé de semenciers et que Sarcelle a au contraire un nombre très élevé de semis en raison du nombre important de semis par semencier (plus de 700) traduisant une forte fructification que nous avons observée en mars-avril 1981.

Pour l'acajou blanc, le même nombre de semenciers à Jules, Sarcelle et la Digue donne des nombres de semis très différents, près de 10 fois plus forts à Sarcelle qu'à la Digue. Les résultats médiocres de Choisy sont dus à un petit nombre de semenciers n'ayant produit qu'un nombre moyen de semis.

Pour le marbri on remarque Choisy dont les semenciers n'ont pratiquement pas donné de semis. Les autres dispositifs avec des nombres de semis par semencier assez voisins donnent des résultats qui sont fonction du nombre de semenciers.

Le palétuvier jaune, représenté toujours par un nombre réduit d'individus (moins de 3 par hectare) a un "pouvoir de régénération » très important, supérieur à celui du bois rouge, et 4 arbres sur une parcelle de 2 ha suffisent à donner 15000 semis sur cette même parcelle (forêt de la Digue).

\subsection{Eflet des différents facteurs expérimentaux}

Le tableau 2 récapitule les résultats de l'analyse de variance faite à partir des données du tableau 1. Cette analyse permet de dégager des tendances plus générales que ne l'autorise le simple examen du tableau 1. On trouve pour chacune des trois variables étudiées : nombre de semis par unité de surface, pouvoir de régénération et nombre de semenciers par unité de surface un effet significatif au seuil de 1 p. 100 des facteurs FORET et ESPÉCE ainsi que de l'interaction entre ces 2 facteurs et un effet significatif au seuil de 5 p. 100 pour le facteur COUPE.

En ce qui concerne le nombre de semenciers, c'est-à-dire la composition initiale des peuplements, tous les niveaux des facteurs ESPECE et FORÊT sont différents, ce qui est normal car les peuplements initiaux n'ont pas la même composition en espèces. Pour le facteur COUPE, les niveaux coupe forte et coupe faible ne sont pas statistiquement différents, conséquence d'une bonne répartition des traitements. Les parcelles témoins, du fait de leur taille inférieure, ont été choisies dans des zones plus riches en espèces précieuses que les autres traitements. 
En ce qui concerne le facteur FORET, c'est Sarcelle qui a le plus de semis parce qu'ayant le plus de semenciers produisant chacun davantage de semis. Il est à noter que l'ordre des forêts est le même pour les 3 variables et que les forêts qui ont le moins de semis ont moins de semenciers produisant chacun peu de semis.

En ce qui concerne le facteur ESPECE, le bois rouge a le plus grand nombre de semis ainsi que le plus fort pouvoir de régénération alors que les semenciers potentiels les plus nombreux sont ceux de gommier. Le nombre moins important de semis de gommier est dû à un pouvoir de régénération assez faible de cette espèce. Pour le marbri et l'acajou blanc on obtient un nombre à peu près identique de semis, mais 10 fois inférieur à celui des essences principales. Le faible nombre d'acajou blanc est compensé par un pouvoir de régénération élevé pour cette espèce.

L'interaction FORET/ESPECE est significative. Ceci est dû essentiellement au bois rouge qui fluctue beaucoup d'une forêt à l'autre alors que les autres especes restent relativement stables.

L'examen du facteur COUPE, nous renseigne sur l'effet des coupes d'ensemencement. Au niveau des placeaux, le nombre de semis est plus élevé pour les coupes faibles que pour les coupes fortes et le témoin qui ne sont statistiquement pas différents. Ces résultats doivent être interprétés en fonction du nombre de semenciers et si on se réfère au pouvoir de régénération c'est-à-dire au nombre de semis par semencicr potentiel on obtient les valeurs les plus fortes et très voisines pour les coupes fortes et faibles tandis que celles du témoin sont près de 2 fois plus perites.

Ces résultats sont d'autant plus intéressants qu'il n'y a pas d'interaction FORÊT/ COUPE ni ESPECE/COUPE et qu'ils sont done globalement valables pour chaque espèce dans chaque forêt.

On peut voir là un effet des coupes d'ensemencement qui ont assuré une meilleure floraison et une fructification accrue ou qui ont permis une meilleure survie des plantules. L'évolution des semis au cours du temps permettra de micux répondre à cette question.

\subsection{Répartition spatiale des semis}

Le tableau 1 donne quelques informations sur la répartition spatiale des semis.

La répartition des semis de gommier est assez uniforme puisque seulement un quart de placeaux n'ont pas de semis et qu'il y a plus de 10 semis dans seulement 10 p. 100 des placeaux. Ceci est confirmé par simple observation car nous n'avons jamais vu de taches de semis de gommier. Le fruit du gommier est une drupe de 2.5 à $3 \mathrm{~cm}$ de diamètre, donc assez grosse, mais est fortement appréciée de certains oiseaux. Ceci, en plus du fait que les gommiers sont toujours présents en nombre important, explique la répartition assez homogène des semis de gommier.

D'après Rollet (1969) la distribution des semis dans les forêts naturelles non perturbées de la Guyane vénézuélienne suit unc loi de Poisson :

$$
X_{n}=\frac{100 x^{11} e^{-x}}{n !}
$$

où $\mathrm{X}_{11}$ est le nombre de placcaux en p. 100 ayant $n$ semis, le nombre de semis par placeau variant de 0 à $\mathrm{N}$, et $\mathrm{x}$ est le nombre moyen de semis par placeau. 


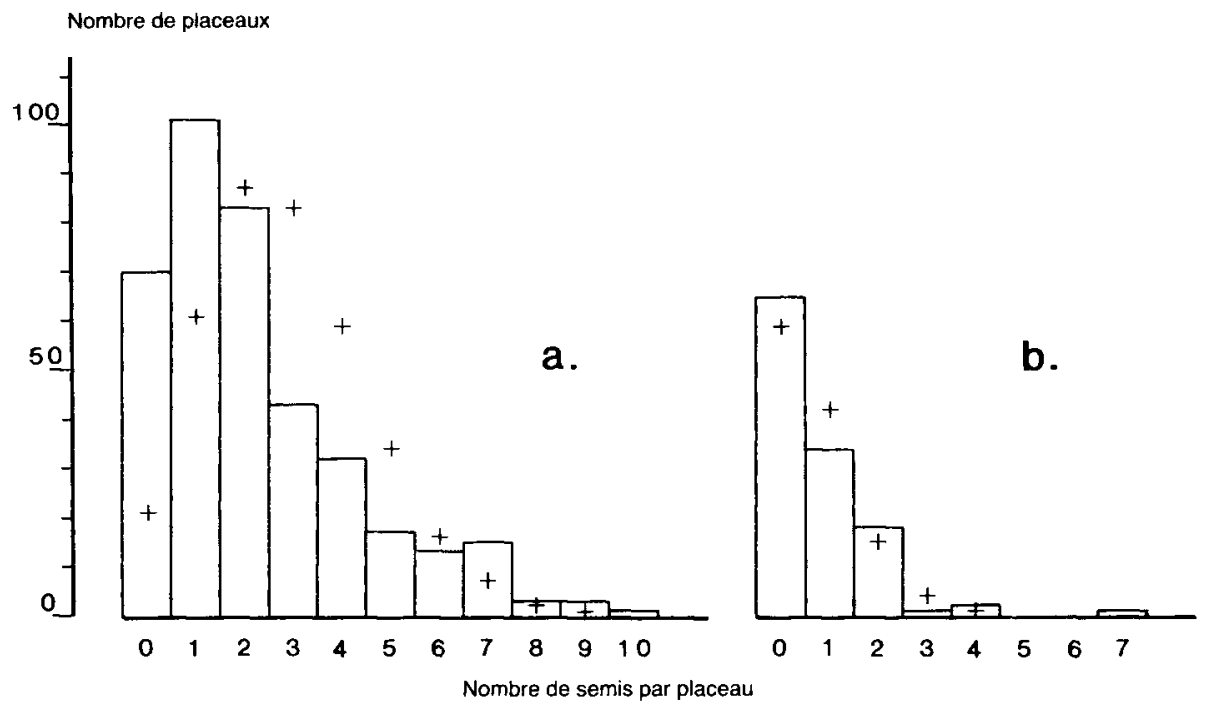

FIG, 1

Histogramme montrant la répartition spatiale des semis de gommier dans ies parcelles éclaircies (a) par comparaison avec le témoin (b). Les signes + indiquent la répartition théorique suivant la loi de Poisson.

Histogram of the spatial distribution of Dacryodes excelsa seedlings in the thinned plots (a) compared with the control plot (b).

The + signs indicate the theoretical distribution according to the Poisson's distribution.

En appliquant cette loi de distribution aux semis de gommier comptabilisés dans le dispositif de Sarcelle en mars 1982 (fig. 1) on obtient pour les 121 placeaux du témoin sous forêt naturelle une distribution proche de la réalité. Pour les 371 placeaux pris dans les 2 types de coupe confondus, on obtient une distribution qui s'éloigne un peu de la loi de Poisson, le nombre de placeaux ayant peu de semis étant dans la réalité plus élevé. Ceci peut être considéré comme un effet des coupes d'ensemencement sur la répartition des semis, deux ans environ après la réalisation de ces coupes.

Pour le bois rouge on n'observe pas la même régularité. D'une parcelle à l'autre le pourcentage de placeaux vides est très variable et peut aller de 20 à $90 \mathrm{p}$. 100 . La distribution de ces semis s'écarte dans tous les cas de la loi de Poisson el on n'a pas pu mettre en évidence un effet des coupes d'ensemencement. La distribution se caractérise en particulier par un nombre important de placeaux ayant plus de 10 semis (entre 10 et 30 p. 100 suivant les dispositifs) et qui traduit la présence de taches de semis. L'existence de ces taches de semis, toujours situées sous les senenciers, est due à une fructification très abondante observée en avril-mai 1981 el à une absence de dissémination des graines qui, bien que moins lourdes que celles de gommier, ne sont cependant pas dispersées par le vent et ne sont pas non plus consommées par les oiseaux. 

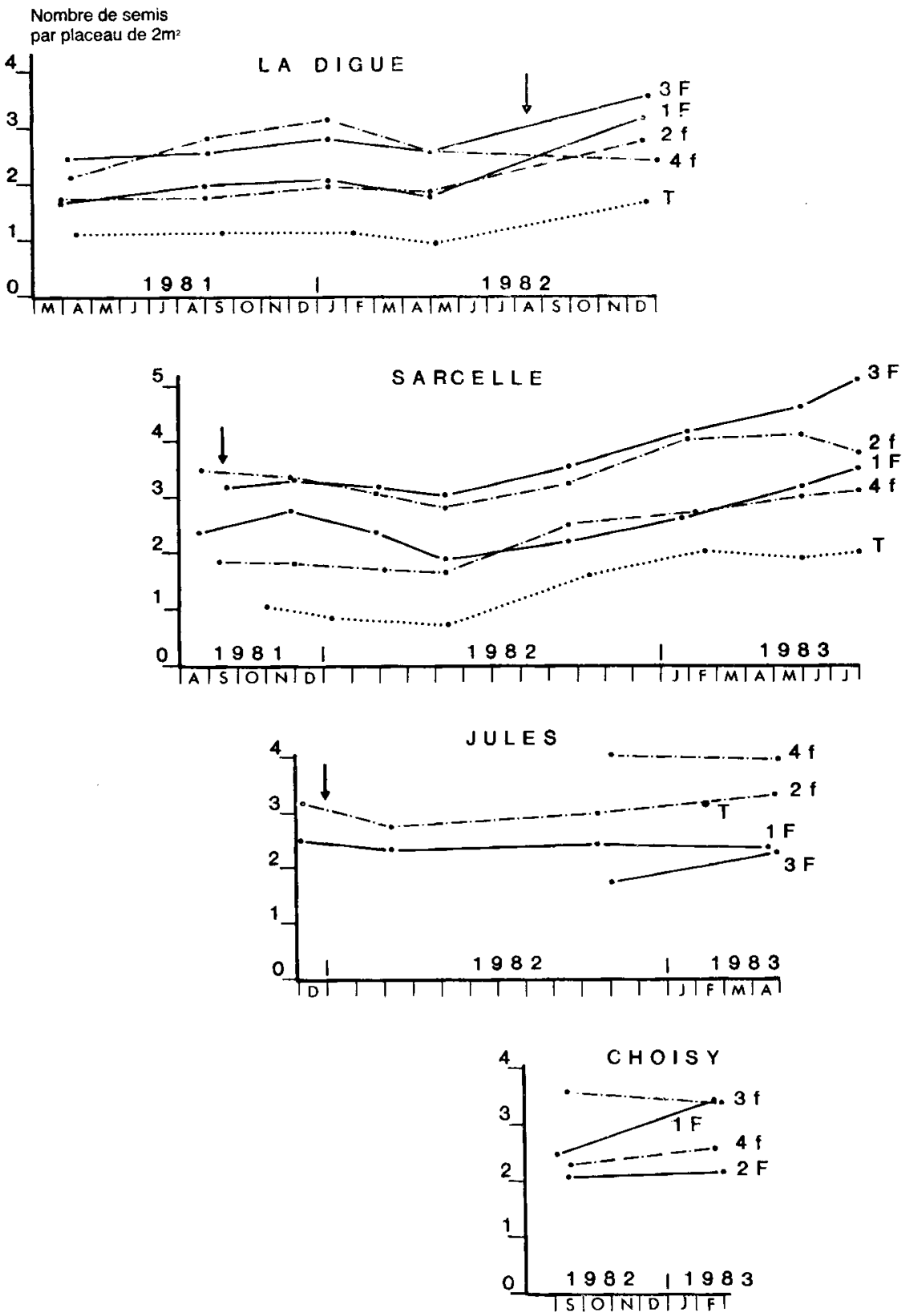

FIG. 2

Evolution au cours du temps de la densité des semis de gommier dans les 4 dispositifs expérimentaux.

Les flèches verticales indiquent les dégagements de semis.

Variation with time of Dacryodes excclsa seedling density at the four sites.

Vertical arrows indicate shrub control operations. 


\subsection{Evolution du nombre de semis au cours du temps et morlalité}

\subsection{Le gommier}

La figure 2 montre l'évolution du nombre de semis entre 1981 et 1983 pour les quatre dispositifs.

La tendance générale est une diminution du nombre de semis pendant la saison sèche, de décembre-janvier à avril-mai, et à une augmentation pendant tout le reste de l'année qui correspond à la saison des pluies.

Le bilan est globalement positif pour la Digue et Sarcelle où le nombre de semis s'accroît d'une année sur l'autre tandis qu'il est pratiquement nul pour Jules et Choisy pour lesquels on ne dispose pas d'observations suffisamment longues. Pour Sarcelle cette augmentation est importante, et la saison sèche 1983 provoque simplement un ralentissement de l'accroissement du nombre de semis. Il y a donc bien une mortalité des semis mais elle reste très faible et ne compromet pas la survie de la régénération.

L'évolution au cours du temps est assez semblable pour toutes les parcelles d'un même dispositif et on ne peut pas mettre en évidence un changement de comportement lié à la nature des coupes d'ensemencement.

\subsection{Le bois rouge carapate}

La fructification importante qui a eu lieu pendant la saison sèche 1981 (fig. 3) s'est traduite par une augmentation du nombre de semis de bois rouge tout au long de la saison des pluies suivante.

A la Digue où il ne restait pratiquement plus de semis de bois rouge après l'exploitation des coupes d'ensemencement, on obtient fin 1981 jusqu'à 50000 semis par hectare dans certaines parcelles. A Sarcelle on arrive même jusqu'à 75000 semis par hectare. Mais dès le début de la saison sèche 1982 la mortalité devient importante dans les 3 dispositifs de la Digue, Sarcelle et Jules et n'est pas compensée par une nouvelle fructification. La mortalité est un peu ralentic pendant la saison des pluies 1982 mais augmente à nouveau pendant la saison sèche 1983 dans le cas de Sarcelle.

On assiste donc à une diminution régulière du nombre de semis de bois rouge, aucune fructification importante n'ayant suivi celle de 1981, sauf peut-être à Jules qui arrive à conserver un nombre de semis à peu près constant. Nous n'avons malheureusement pas d'informations sur la fréquence de fructification du bois rouge et il est donc impossible de prévoir l'évolution future de cette régénération.

La figure 3 ne permet pas de mettre en évidence un effet quelconque des coupes d'ensemencement sur l'évolution du nombre de semis.

\subsection{L'acajou blanc}

L'examen de la figure 4 montre une certaine stabilité dans le nombre de semis d'acajou blanc, à l'exception toutefois de la parcelle 1 de Jules naturellement riche en acajou blanc ayant fait l'objet d'une fructification importante mais vite perdue que l'on ne retrouve ni à Sarcelle ni à la Digue. 

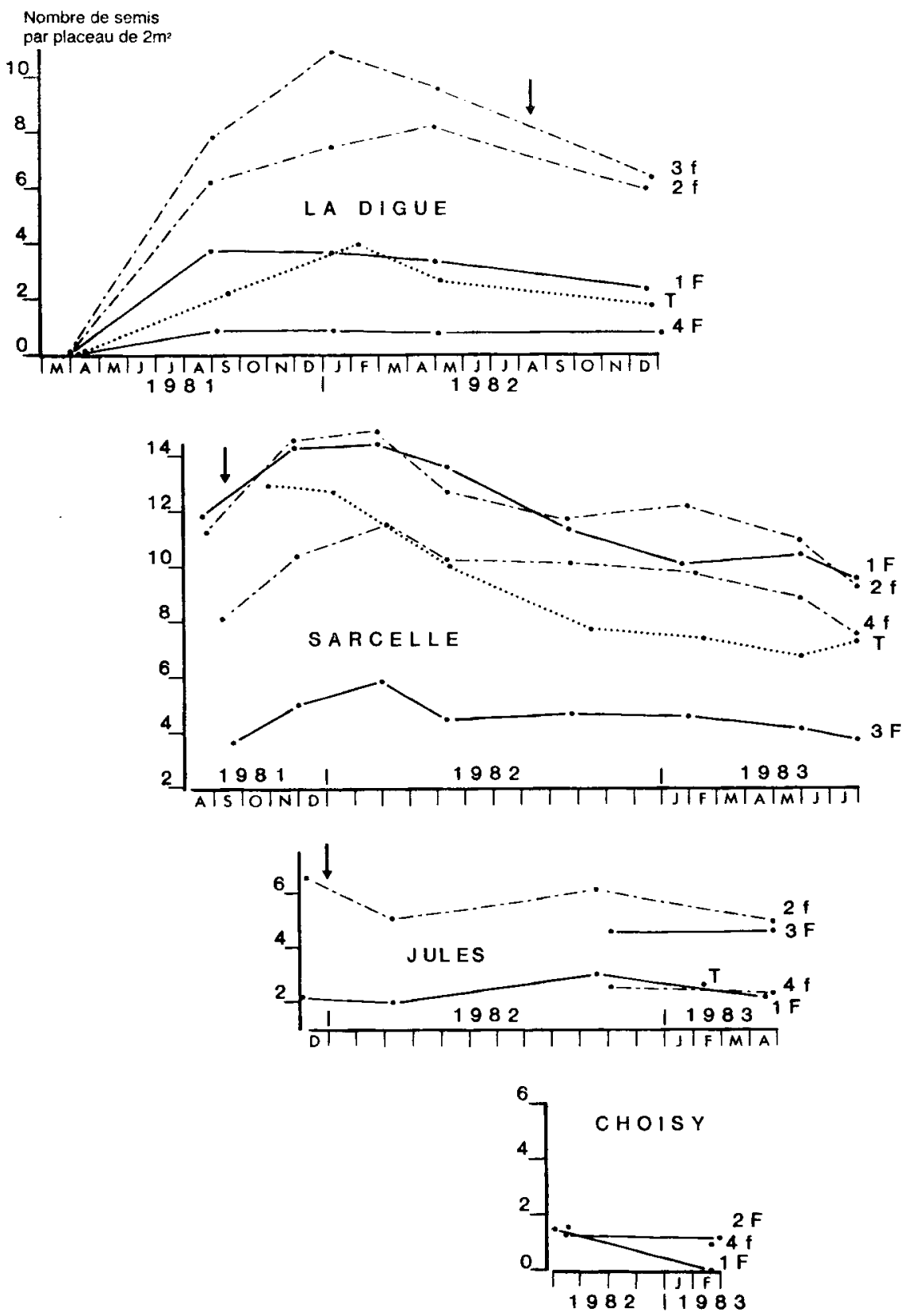

FIG. 3

Evolution au cours du temps de la densité des semis de bois rouge dans les 4 dispositifs expérimentaux.

Les flèches verticales indiquent les dégagements de semis.

Variation with time of Amanoa caribaea seedling density at the four sites. Vertical arrows indicate shrub control operations. 
Nombre de semis

par placeau de $2 m^{2}$

L A D I G UE
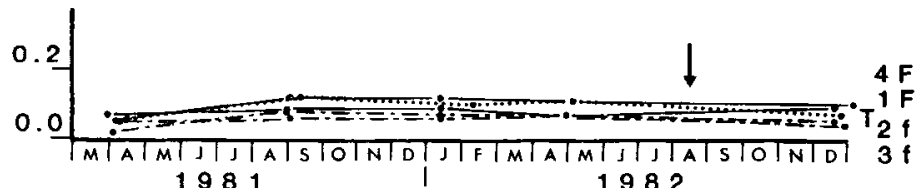

1981

1982
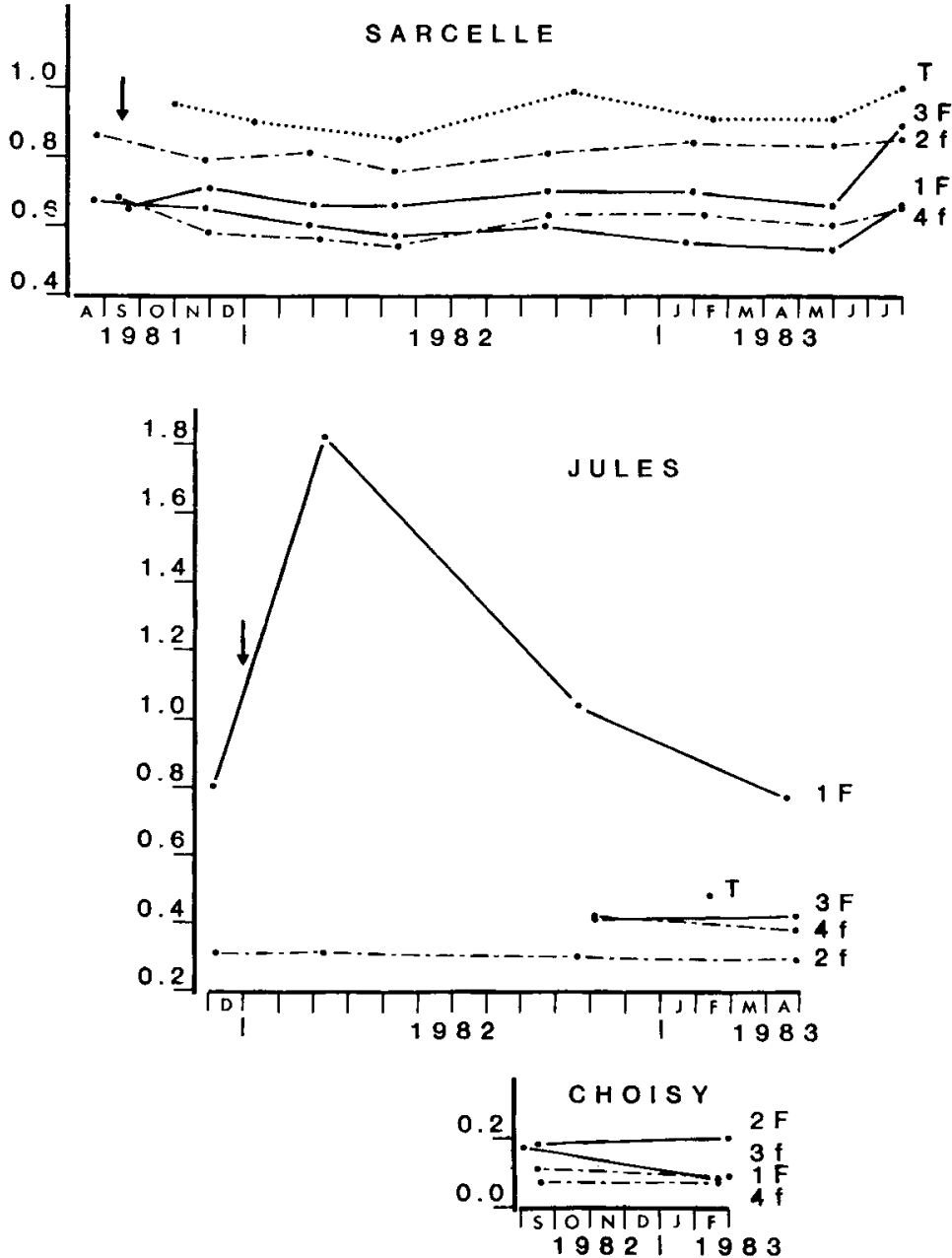

FIG. 4

Evolution au cours du temps de la densité des semis d'acajou blanc dans les 4 dispositifs expérimentaux.

Les flèches verticales indiquent les dégagements de semis.

Variation with time of Simaruba amara seedling density at the four sites. Vertical arrows indicate shrub control operations. 
Cette stabilité est presque parfaite à la Digue avec une densité de scmis très faible et dont la moitié sont des préexistants. Elle l'est aussi à peu près à Sarcelle où l'on note milieu 1983 une tendance à l'augmentation du nombre de semis.

\subsection{Le marbri}

On note pour le marbri une forte augmentation du nombre de semis à la fin de 1981 (fig. 5). Cette fructification ne se répète pas en 1982 aussi le bénéfice de la
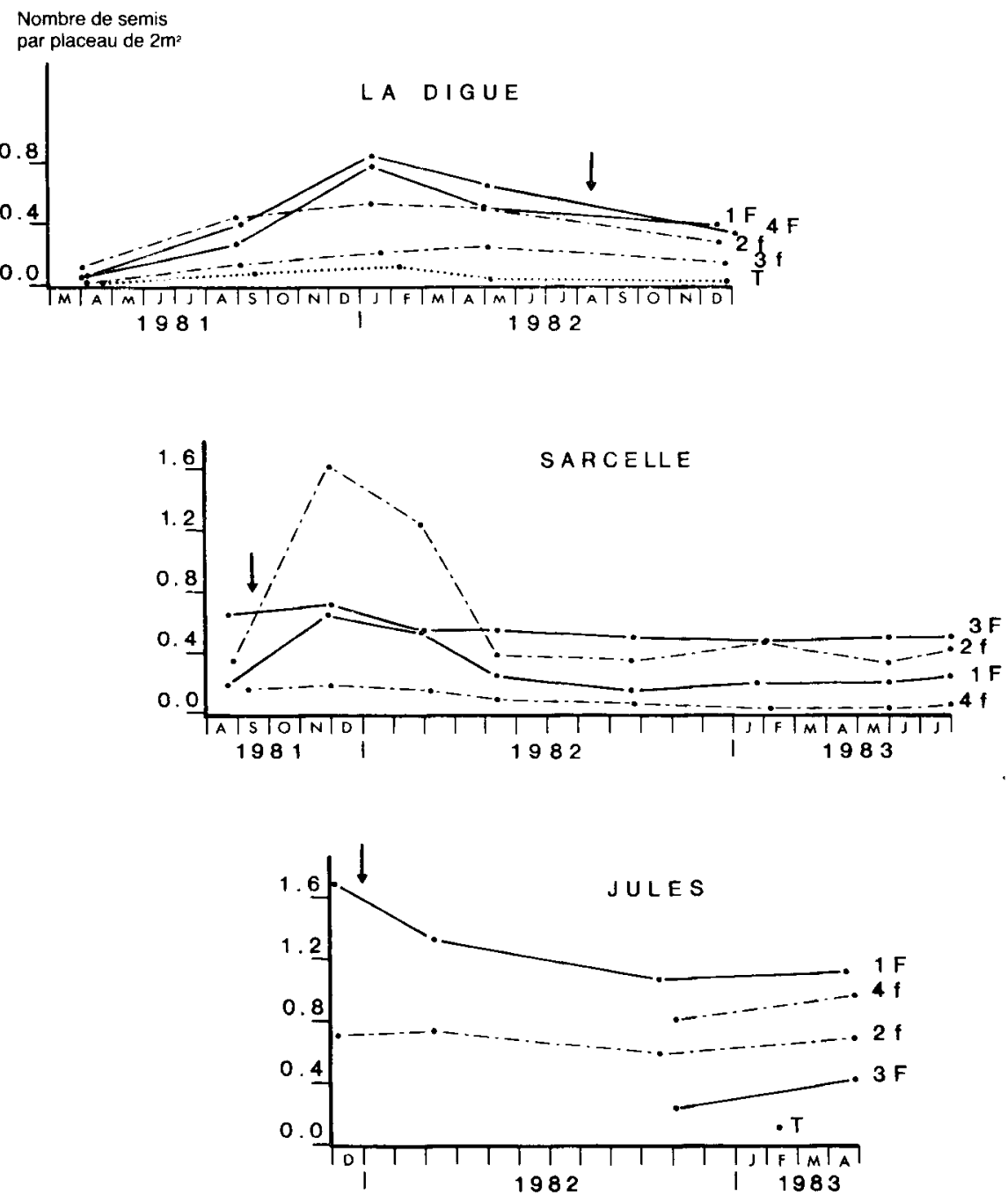

Fig. 5

Evolution au cours du temps de la densité des semis de marbri dans les 4 dispositifs expérimentaux.

Les flèches verticales indiquent les dégagements de semis.

Variation with time of Richeria grandis seedling density at the four sites.

Vertical arrows indicate shrub control operations. 
fructification de 1981 est pratiquement nul dans le cas de Sarcelle tandis qu'à la Digue le gain est positif par rapport aux quelques préexistants qui restaient après les coupes d'ensemencement.

\section{Croissance des semis d'espèces précieuses}

Les mesures de croissance en hauteur sont le complément logique à notre étude de régénération naturelle car elles permettent d'une part de déterminer l'aptitude à la croissance des semis dans les conditions particulières des coupes donc leur capacité à échapper à la concurrence herbacée et arbustive, et d'autre part d'obtenir des informations sur le tempérament des différentes espèces vis-à-vis de la lumière.

\subsection{Mesure de la croissance en hateur}

Ces mesures ont été faites entre novembre 1981 et avril 1983 sur des périodes de 13 mois à la Digue, 15 mois à Sarcelle et 17 mois à Jules (sauf parcelles 3 et 4 : 4 mois). Pour plus de facilité, les accroissements en hauteur ont été ranenés à une durée annuelle et sont rassemblés sur la figure 6 .

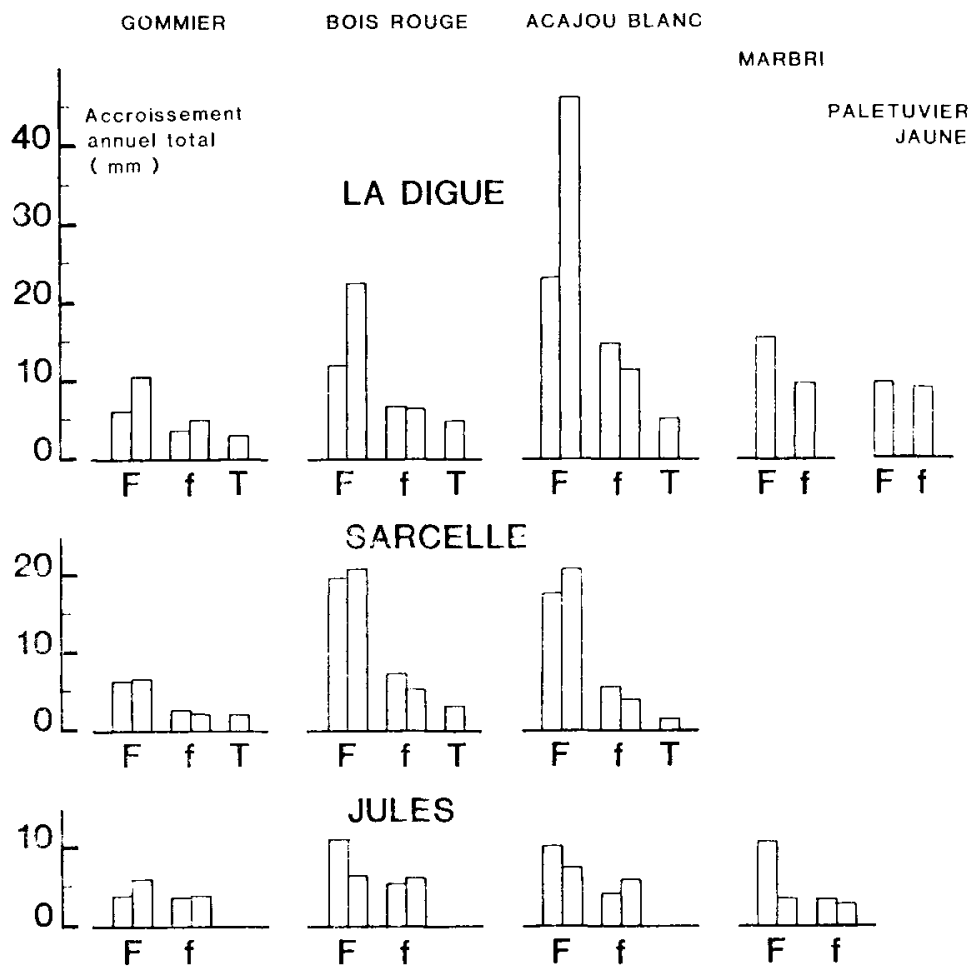

Fig. 6

Accroissement annuel total en hauteur des semis des différentes espèces dans les parcelles fortement éclaircies $(F)$, faiblement éclaircies $(f)$ et témoin $(T)$ des dispositifs de la Digue, Sarcelle et Jules.

Mean annual total height increment of seedlings of the various species for the 3 treatments : heavily felled plots $(F)$, lightly felled plots $(f)$ and control plot $(T)$ at 3 sites (La Digue, Sarcelle and Jules). 
La croissance en hauteur du gommier reste toujours faible comparée à celle des autres espèces. Cependant l'influence des coupes est toujours favorable. Ainsi les maximums d'accroissement se situent dans les coupes fortes de Sarcelle et de la Digue et à un degré moindre dans celles de Jules (respectivement $6,5 \mathrm{~cm}, 8,2 \mathrm{~cm}$ et $4,9 \mathrm{~cm})$ tandis que les minimums d'accroissement se situent dans les témoins $(2,5 \mathrm{~cm}$ en moyenne).

L'accroissement en hauteur du bois rouge peut atteindre des valeurs assez fortes : souvent plus du double des accroissements mesurés sur le gommier. Cette espèce semble réagir aussi très favorablement à l'ouverture du couvert. Les maximums d'accroissement sont observés dans les coupes de Sarcelle, la Digue et Jules (respectivement $20,3 \mathrm{~cm}, 17,2 \mathrm{~cm}$ et $8,7 \mathrm{~cm}$ ) tandis que les valeurs intermédiaires sont observées dans les coupes faibles (respectivement $6,3 \mathrm{~cm}, 6,7 \mathrm{~cm}$ et $5,9 \mathrm{~cm}$ ) et les plus faibles dans les témoins (respectivement $3,1 \mathrm{~cm}, 5,0 \mathrm{~cm}$ pour Sarcelle et la Digue).

L'acajou blanc est l'espèce qui réagit le plus favorablement aux coupes bien que les différences entre parcelles soient souvent assez importantes. Pour Sarcelle et la Digue, les accroissements en hauteur sont respectivement de $19,5 \mathrm{~cm}$ et $34,8 \mathrm{~cm}$ pour les coupes fortes, $4,9 \mathrm{~cm}$ et $13,2 \mathrm{~cm}$ pour les coupes faibles et pour les témoins $1,6 \mathrm{~cm}$ et $5,2 \mathrm{~cm}$. Les accroissements à Jules sont de $8,9 \mathrm{~cm}$ pour les coupes fortes et $5,2 \mathrm{~cm}$ pour les coupes faibles.

Les autres espèces précieuses réagissent aussi favorablement à une ouverture du couvert. C'est le cas du marbri pour lequel on observe des accroissements en hauteur de $7,2 \mathrm{~cm}$ à Jules et $15,6 \mathrm{~cm}$ à la Digue pour les coupes fortes et de $3,2 \mathrm{~cm}$ à Jules et $9,7 \mathrm{~cm}$ à la Digue pour les coupes faibles. C'est le cas également, mais dans une moindre mesure, du palétuvier jaune qui, à la Digue, a un accroissement de $9,8 \mathrm{~cm}$ sous coupe forte et $9,1 \mathrm{~cm}$ sous coupe faible.

\subsection{Effet des différents facteurs expérimentaux}

L'analyse de variance montre un effet significatif des 3 facteurs étudiés (tabl. 3). Les accroissements, total et relatif, sont plus importants à la Digue qu'à Sarcelle. L'acajou blanc pousse plus rapidement que le bois rouge en valeur absolue, mais les accroissements relatifs sont inversés car, nous l'avons vu, il y a davantage de préexistants parmi les acajous blancs et ils sont de taille supérieure aux bois rouges. Le gommier pousse moins vite que les 2 autres espèces.

Les régénérations poussent plus rapidement dans les coupes fortes que dans les coupes faibles et le témoin. Bien que l'analyse ne montre pas de différences significatives entre les coupes faibles et le témoin on a pu voir (fig. 6) que les accroissements sont toujours légèrement plus faibles dans le témoin. L'examen de l'interaction COUPE/ESPECE, significative au scuil de 1 p. 100, montre que les espèces réagissent de manière différente aux coupes bien que globalement il y ait un effet COUPE bien marqué. En réalité, l'effet des coupes va bien dans le même sens pour toutes les espèces; seulement, cet effet est beaucoup plus fort pour l'acajou blanc que pour les autres. Si on se réfère à l'accroissement total dans les témoins, la mise en lumière dans les coupes fortes multiplie la croissance en hauteur par 3 pour le gommier, par 4 pour le bois rouge et par 9 pour l'acajou blanc. Le paragraphe suivant permettra de préciser l'effet des coupes, donc de la lumière sur la croissance des semis. 


\section{TABLEAU 3}

Effet des facteurs expérimentaux sur la croissance des semis : analyse de variance et comparaison des moyennes.

Effect of controlled factors on seedling growth : variance analysis and mean multiple comparisons.

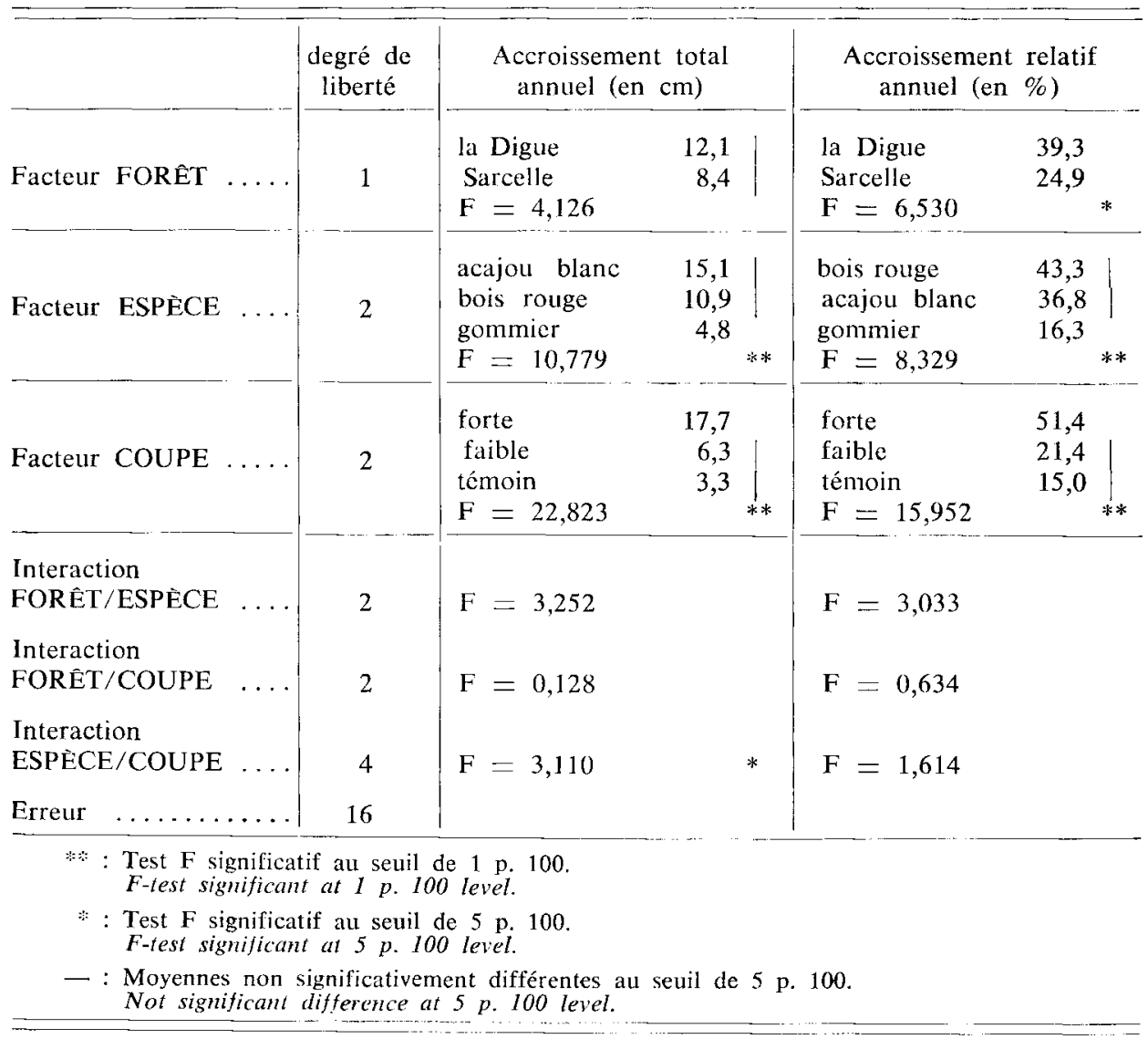

\subsection{Influence du microclimat lumineux sur la croissance}

Les mesures de perméabilité relative du feuillage au rayonnement solaire faites par photographies hémisphériques dans le dispositif de Sarcelle donnent une estimation moyenne du microclimat lumineux dans chaque parcelle.

L'accroissement total en hauteur a été relié à la perméabilité relative pour chacune des trois espèces principales (fig. 7). C'est l'acajou blanc qui réagit le plus favorablement à la lumière, suivi du bois rouge et du gommicr. Les coefficients de corrélation sont bons statistiquement, mais il ne faut pas trop y attacher d'importance en raison du nombre réduit de couples de mesures. 
En raison de la relation existant entre la perméabilité relative et la surface terrière (DuckeY \& LABBÉ, 1985) nous avons relié l'accroissement total à la surface terrière pour les dispositifs de Sarcelle et la Digue et nous avons trouvé une relation linéaire entre l'accroissement total et l'inverse de la surface terrière (fig. 7). Les coefficients de corrélation obtenus sont du même ordre, sinon meilleurs, que ceux obtenus avec la perméabilité relative. Le rayonnement solaire a dans tous les cas un effet positif. On n'a donc pas encore atteint l'optimum de croissance des différentes espèces. On peut penser que des coupes plus fortes auraient permis une meilleure croissance des semis, mais ce n'est pas certain car il faut tenir compte de la concurrence des lianes et des plantes herbacées qui devient rapidement très importante.

\section{SARCELLE}
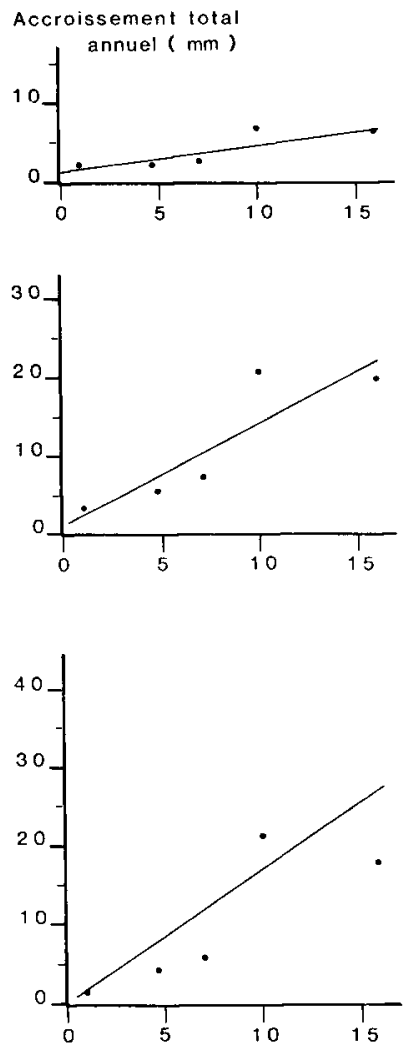

Perméabilité relative en ( $\%$ )
GOMMIER
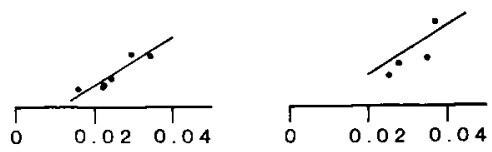

BOIS ROUGE
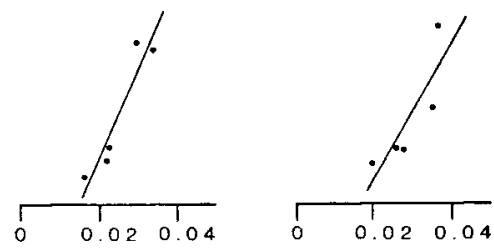

ACAJOU BLANC

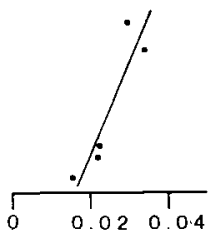

Inverse de la surface terrière $\left(\right.$ ha. $\left.\mathrm{m}^{-2}\right)$

FIG. 7

Effets de la perméabilité relative au rayonnement solaire et de la surface terrière sur l'accroissement total annuel en hauteur des différentes espèces dans les dispositifs de Sarcelle et la Digue.

Effects of relative permeability to solar radiation and of total basal area on annual total height increment for various species at 2 sites (Sarcelle and La Digue). 


\section{TABLEAU 4}

Evolution saisonnière de la croissance en hauteur dans le dispositif de Sarcelle, en relation avec différents paramètres climatiques.

Seasonal variation of height growth related to various climatic factors at Sarcelle.

\begin{tabular}{|c|c|c|c|c|c|c|}
\hline \multirow[b]{2}{*}{ Périodes } & \multirow{2}{*}{$\begin{array}{l}\text { Parcelles } \\
\text { et coupes }\end{array}$} & \multicolumn{3}{|c|}{ Croissance relative $\mathrm{C} \%$ ) } & \multirow{2}{*}{$\begin{array}{c}\text { Température } \\
\text { maximale } \\
\text { TM ("C) }\end{array}$} & \multirow{2}{*}{$\begin{array}{l}\text { Précipitation } \\
\quad P(\mathrm{~mm})\end{array}$} \\
\hline & & $\begin{array}{l}\text { Gom- } \\
\text { mier }\end{array}$ & $\begin{array}{l}\text { Bois } \\
\text { rouge }\end{array}$ & $\begin{array}{c}\text { Acajou } \\
\text { blanc }\end{array}$ & & \\
\hline \multirow{5}{*}{$\begin{array}{l}\text { Novembre- } \\
\text { décembre } \\
1981\end{array}$} & 1 forte & 4,35 & 9,57 & 8,95 & 26,5 & \multirow{5}{*}{907} \\
\hline & 2 faible & 3,83 & 4,74 & 6,51 & 26,1 & \\
\hline & 3 forte & 6,44 & 9,71 & 6,49 & 25,7 & \\
\hline & 4 faible & 5,27 & 2,14 & 4,73 & 25,8 & \\
\hline & Témoin & 2,38 & 1,98 & 2,42 & 25,1 & \\
\hline \multirow{5}{*}{$\begin{array}{l}\text { Janvier- } \\
\text { février } \\
1982\end{array}$} & 1 forte & 1,83 & 6,74 & 4,20 & 25,1 & \multirow{5}{*}{291} \\
\hline & 2 faible & 1,38 & 4,58 & 1,99 & 24,7 & \\
\hline & 3 forte & 2,50 & 7,27 & 2,79 & 24,7 & \\
\hline & 4 faible & 2,80 & 6,23 & 1,95 & 24,7 & \\
\hline & Témoin & 2,46 & 4,69 & 2,24 & 24,1 & \\
\hline \multirow{5}{*}{$\begin{array}{l}\text { Mars-avril } \\
1982\end{array}$} & 1 forte & 5,13 & 12,28 & 5,31 & 26,2 & \multirow{5}{*}{320} \\
\hline & 2 faible & 5,73 & 7,06 & 3,71 & 25,6 & \\
\hline & 3 forte & 5.59 & 11,54 & 4,79 & 26,2 & \\
\hline & 4 faible & 2,45 & 6,39 & 2,39 & $\begin{array}{l}20,2 \\
25,2\end{array}$ & \\
\hline & Témoin & 1,69 & 1,87 & 1,10 & 25,0 & \\
\hline
\end{tabular}

\subsection{Variation saisonnière de la croissance}

Le tableau 4 montre, pour Sarcelle, de grandes différences de croissance des semis suivant les périodes de mesure. L'accroissement en janvier-février est, à 2 exceptions près, beaucoup plus faible que pendant les 2 autres périodes. On a relié ces accroissements, pour chaque espèce, à la moyenne des températures maximales de la période considérée. Nous obtenons :

- Pour le gommier

- Pour le bois rouge

- Pour l'acajou blanc

$$
\begin{aligned}
& \mathrm{C}(\mathrm{p} .100)=1,76 \mathrm{TM}-41,18 \quad(\mathrm{r}=0,73) \\
& \mathrm{C}(\text { p. } 100)=3,21 \text { TM }-73,94 \quad(r=0,78) \\
& \mathrm{C}(\mathrm{p} .100)=2,61 \text { TM }-62,15 \quad(\mathrm{r}=0,83)
\end{aligned}
$$

Ces relations englobent d'une part les différences de températures maximales observées dans chaque parcelle pendant la même période et donc liées aux coupes, et d'autre part les différences de températures maximales d'une période à l'autre, ces différences étant d'ordre saisonnier.

On peut aussi penser que le ralentissement de la croissance est lié à la pluviométrie. C'est en partie vrai puisque l'on passe de $907 \mathrm{~mm}$ de pluie pour novembre et décembre 1981 à $291 \mathrm{~mm}$ en janvier et février 1982. Mais, pour une qualité de 
précipitations à peu près identique en mars et avril $1982(320 \mathrm{~mm})$ la croissance atteint un niveau souvent supérieur à celui de novembre et décembre 1981 .

Il est très difficile de séparer l'effet des différents facteurs climatiques d'autant plus qu'en Guadeloupe la saison sèche correspond à peu près à la période pendant laquelle la température et la photopériode sont les plus basses. Des études complémentaires seraient d'ailleurs nécessaires pour connaître les exigences photopériodiques et les rythmes internes de chaque espèce.

\section{Discussion et conclusion}

Les observations faites, d'une part sur les peuplements adultes et leur composition juste avant la mise en régénération, d'autre part sur les semis, leur évolution au ccurs du temps et leur croissance, permettent de se faire une première idée du comportement de ces espèces. Nous essayerons donc dans un premier lemps de synthétiser le comportement espèce par espèce, puis dans un second temps de replacer le comportement individuel des espèces dans la dynamique d'évolution de la forêt hygrophile et enfin d'en tirer les premières conclusions sylvicoles.

\subsection{Comportement des différentes espèces}

Les espèces forestières qui nous intéressent sont pratiquement toutes endémiques de l'Are Antillais à l'exception de Simaruba amara et de Symphonia globulifera que l'on retrouve en Amazonie. Elles ont été de ce fait très peu étudiées, surtout sur le plan écologique et il n'est malheureusement pas possible d'étayer nos conclusions par des observations faites par d'autres chercheurs.

\subsection{Comportement da gommier}

Le gommier est l'espèce précieuse la plus fréquente de la forêt hygrophile et représente en moyenne 20,7 p. 100 du nombre de tiges de la $4^{\circ}$ série d'Aménagement ; c'est aussi la plus fréquente de toutes les espèces de la strate dominante ou émergente de cette forêt. Le gommier a donc une position importante et il est intéressant de se demander pourquoi il a acquis cette position et comment il s'y maintient.

Tout d'abord nous avons vu que les semis sans être très abondants sont régulièrement disséminés (seulement 25 p. 100 de placeaux sans semis), le faible «pouvoir de régénération " du gommier, ainsi que la taille des graines étant compensés par le rôle des oiseaux dans la dissémination. Le nombre de semis a tendance à augmenter régulièrement d'une année sur l'autre en dépit d'une mortalité faible mais tout de même existante.

L'effet des coupes est sensible sur le «pouvoir de régénération » du gommier qui a produit davantage de semis par semencier dans les coupes d'ensemencement que dans le témoin. Les semis de gommier réagissent aussi favorablement aux coupes et donc à la mise en lumière et leur croissance en hauteur est plus élevée sous coupe forte que sous coupe faible et témoin. Le gommier est aussi l'essence dont la croissance est la moins accélérée par la mise à la lumière. 
Toutes ces observations nous montrent que le gommier a le comportement caractéristique d'une espèce d'ombre ayant une certaine capacité à attendre, sous couvert, des conditions plus favorables.

\subsection{Comportement du bois rouge carapate}

Le bois rouge carapate vient directement après le gommicr du point de vue de son importance avec $12,6 \mathrm{p} .100 \mathrm{du}$ nombre de tiges de la 4" série. C'est aussi un arbre de la strate dominante où il est en mélange pied à pied avec le gommier bien que l'on puisse trouver occasionnellement des bouquets de bois rouge. Mais les analogies avec le gommier s'arrêtent là et il y a de nombreuses différences entre ces 2 espèces.

Tout d'abord on a vu que les semis sont très nombreux et surtout irrégulièrement répartis (le nombre de placeaux vides varie de 20 à 90 p. 100 mais dans d'autres il peut y avoir jusqu'à 100 semis par mètre carré), ceci étant confirmé par l'existence de véritables brosses de semis principalement sous les semenciers. La fructification peut donc être importante (mais avec quelle fréquence ?) tandis que la dissémination est très faible, les graines étant trop lourdes pour être transportées par le vent et n'étant pas ingérées par les oiseaux.

On ne peut pas mettre en évidence de différences de densité de semis entre les différents types de coupes, mais comme pour le gommier on note un effet bénéfique des coupes sur le «pouvoir de régénération» du bois rouge. De plus, on enregistre des fluctuations importantes du nombre de semis au cours de l'année : le bois rouge est donc beaucoup plus sensible aux conditions extérieures que le gommier. La mortalité est assez importante surtout pendant la saison sèche. Nous avons effectivement vu que, sous forêt naturelle, des taches de semis pouvaient disparaître entièrement d'une année à l'autre : il faut une certaine quantité de lumière, plus importante que celle du sous-bois de la forêt naturelle pour permettre leur survie.

La croissance des semis est influencée très favorablement par la lumière et à quantité égale de lumière, la croissance en hauteur du bois rouge est 2 fois plus importante que celle du gommier. Ceci favorise nettement le bois rouge au cours de la compétition très dure qui s'installe entre les semis et la végétation concurrente pendant la phase de régénération.

Le bois rouge apparaît donc comme une espèce capable de fructifier très abondamment à un moment donné, le succès éventuel de la régénération ainsi initiée étant dû essentiellement à un apport de lumière important, naturel sous forme de chablis ou artificiel dans le cas d'une coupe. Si ces conditions ne sont pas remplies les semis disparaîtront très rapidement. C'est un comportement qui rappelle celui des essences de lumière avec des nuances cependant, puisque le bois rouge supporte quand même des ombrages assez importants et qu'il ne s'installe pas directement en plein découvert.

\subsection{Comportement de l'acajou blanc}

L'acajou blanc est seulement la $4^{\circ}$ espèce précieuse de par sa fréquence dans les peuplements de la forêt hygrophile. C'est néanmoins une espèce facilement identifiable et partout présente dans les régénérations ce qui laisse souvent penser qu'elle est très abondante alors qu'on ne rencontre que rarement plus de 3000 semis par hectare et que le nombre de semenciers oscille autour de 10 par hectare. 
Les semis sont à pcu près régulièrement répartis avec une préférence toutefois pour les clairières, cette répartition étant facilitée par la légèreté des graines et par un complément de dissémination par les oiseaux. La croissance des semis est aussi très fortcment influencée par la mise à la lumière et est comparable à celle du bois rouge. Par ailleurs, nous avons pu constater la présence d'acajou blanc dans les lisières et en bordure des routes forestières ce qui indique la possibilité pour cette espèce de vivre en plein découvert.

Cette espèce apparâit donc comme une espèce de lumière, plus encore que le bois rouge mais qui est cependant présente dans la forêt hygrophile en raison de la fréquence des chablis dans cette forêt.

\subsection{Comportement des autres espèces précieuses}

Nous ne disposons que de peu d'informations sur les autres espèces qui sont très peu abondantes et que nous n'avons pas toujours pu observer.

Le marbri est présent dans tous les dispositifs en plus grand nombre que l'acajou blanc (c'est la $3^{\text {c }}$ espèce précicuse avec $8,5 \mathrm{p} .100 \mathrm{du}$ nombre de tiges en $4^{\prime \prime}$ série). C'est un arbre de la strate moyenne que l'on trouve à l'état isolé mais aussi plus rarement en peuplements presque purs.

Les semis sont en nombre variable dans les coupes mais beaucoup moins nombreux sinon absents dans les parcelles témoins. Ils réagissent très bien aux différents types de coupe done à la lumière dans les peuplements où ils ont été observés. Leur croissance est alors similaire à celle du bois rouge ou de l'acajou blanc. Comme nous n'avons que peu d'observations sur cette espèce, il est difficile d'être très catégorique mais son comportement révèle un tempérament voisin de celui du bois rouge ou de l'acajou blanc, plus proche peut-être de celui de l'acajou blanc si on pense à la présence de cette espèce en lisière ou en bordure de routes forestières.

Le palétuvier jaune est une espèce plus rare que l'on trouve principalement dans les bas de versants mouilleux et qui n'est pas présente de ce fait dans tous les dispositifs. Même quand elle est présente, les semis peuvent être absents (cas des parcelles 1 ct 2 de Jules) ou très abondants (plus de 6000 par hectare dans la parcelle 4 de la Digue). Les semis réagissent aussi favorablement à la lumière mais leur croissance reste faible, voisine de celle du gommier. On est tenté de la considérer comme une espèce d'ombre mais ses conditions particulières d'habitat en font un peu une espèce à part.

On rencontre enfin dans les parcelles expérimentales du mauricif et du bois doux chypre. Mais en raison de leur nombre très réduit et de la difficulté de reconnaissance des stades juvéniles des semis, ils n'ont pas pu être observés.

\subsection{Conséquences sur la composition de la forêt naturelle}

Le comportement de chaque espèce lui assigne un statut particulier au sein de la forêt naturelle.

A ce statut correspondent une place et une importance qui évoluent tout au long des cycles sylvigénétiques (OLDEman, 1972) qui régissent la vie de la forêt. L'apparition de trouées déclenche une nouvelle phase de régénération naturelle. 
L'ampleur de cette régénération et les espèces concernées sont fonction de la perturbation créée (Ashton, 1978; Whitmore, 1978; Rollet, 1983). On peut essayer d'appliquer ces principes dans notre cas particulier.

Tout d'abord le gommier avec son tempérament d'essence d'ombre dont les semis supportent bien les conditions du sous-bois forestier et peuvent se développer avec des apports énergétiques faibles constitue la base du peuplement adulte : on a vu que c'était l'espèce la plus fréquente y compris des espèces diversus. II se régénère au niveau des petits chablis ou à proximité des chablis plus importants.

Le bois rouge quant à lui nécessite davantage de lumière pour se maintenir et prospérer dans le sous-bois. Il se régénère donc à la faveur des chablis plus importants (chute des arbres émergents, cyclones de moyenne importance). Sa grande capacité de fructification et de régénération en font un arbre assez fréquent dans la forêt naturelle.

L'acajou blanc avec son tempérament d'essence de lumière se développe dans les chablis très importants, vraisemblablement causés par les très forts cyclones. I1 est alors en compétition directe avec les espèces pionnières telles que la côtelette blanche (Miconia mirabilis) et il doit à sa bonne croissance en hauteur de pouvoir survivre. C'est pourquoi on le retrouve à l'état disséminé dans les peuplements adultes où il est alors le vestige d'une phase de rajeunissement de la forêt. D'anciens stades de rajeunissement existent dans nos dispositifs expérimentaux où on trouve encore des groupes de côtelettes blanches dépérissantes. C'est souvent à l'ombre de ce couvert d'espèces pionnières que se sont développées d'autres espèces comme le marbri, le mauricif, le bois doux chypre et bien évidemment le bois rouge qui profitent aussi de ces conditions favorables.

D'autres espèces enfin prennent localement une plus grande importance en raison de leur habitat spécifique. C'est le cas du palétuvier jaune, nous venons de le voir, ou du résolu que nous n'avons rencontré que rarement car il vit à proximité immédiate des petits cours d'eau.

Pour être plus complète, notre analyse aurait dû comprendré l'ensemble des espèces de la forêt naturelle mais il aurait fallu pour cela inclure à notre étude une dizaine d'espèces supplémentaires.

\subsection{Premières conclusions sylvicoles}

Nous avons vu que dans les conditions naturelles la régénération de la forêt se fait de manière incontrôlée par «catastrophes». Dans le cadre de la sylviculture, le forestier dispose, par le biais des coupes, d'un très large éventail de possibilités pour modifier le couvert et pour contrôler cette régénération à sa guise, en fonction des peuplements qu'il souhaite obtenir. Le «Tropical Shelterwood system » (Rosevear \& Lancaster, 1953) était inspiré de cette idée de même que la méthode que nous utilisons et qui est très proche de la précédente. De fait la sylviculture des peuplements mélangés devient vite très complexe ce qui explique que les meilleurs résultats soient obtenus dans les forêts naturellement riches en quelques espèces (CATıNOT, 1974) ce qui est le cas de la Guadeloupe.

Dans la forêt hygrophile de Guadeloupe, l'observation initiale des semenciers présents et si possible des semis installés permettra au forestier de s'orienter dans deux voies différentes : 
- En l'absence de semenciers de bois rouge et de régénération importante d'essences de lumière (acajou blanc et marbri), les nettoiements et coupes devront se faire en faveur du gommier, essence principale du peuplement en place dont la régénération est presque assurée. Ces coupes resteront toujours faibles ce qui évitera, d'une part, la concurrence d'essences héliophiles inintéressantes et d'autre part, des interventions nombreuses en dégagements. La durée de mise en régénération risque cependant d'être assez longue mais il est encore trop tôt pour la définir.

- En présence de semenciers de bois rouge et, si possible après acquisition d'une régénération importante en essences précieuses (taches de semis de bois rouge mais aussi présence d'acajou blanc, de marbri et éventuellement de palétuvier jaune), on pourra intervenir de manière dynamique pour favoriser la régénération. Ces interventions comprendront des coupes fortes qui assureront l'avenir du bois rouge et des autres essences de lumière. La durée de mise en régénération sera certainement cette fois-ci beaucoup plus courte mais le nombre de dégagements nécessaires risque d'être plus élevé. Le problème des dégagements est évidemment fondamental au point de vue économique (Schulz, 1967) et il est impératif de trouver un compromis entre une bonne croissance des semis et un envahissement herbacé excessif.

Comme les peuplements sont en général très mélangés, une solution intermédiaire entre ces 2 méthodes pourrait consister à mettre à la lumière les taches de semis d'essences à tendance héliophile là où elles existent, et à conserver par des coupes faibles les semis de gommier qui sont de toute façon présents, là où ces taches n'existent pas. Des dégagements pourraient avoir lieu en même temps que ces passages en coupe.

Nous venons alors de définir une orientation vers la futaie jardinée pour ces peuplements. Cette solution serait sans doute très satisfaisante car elle aurait l'avantage, d'une part, de conserver l'ensemble des différentes essences précieuses, on pourrait d'ailleurs conserver un certain pourcentage d'espèces diverses, et d'autre part, de maintenir une structure de type jardiné, très différente de la futaie régulière, et plus résistante aux risques naturels d'érosion et de cyclones.

Il faut toutefois se garder de généraliser nos conclusions et insister sur le fait qu'une telle sylviculture intensive est envisageable seulement dans des forêts naturellement riches en quelques espèces précieuses ainsi que nous l'avons dit et répété tout au long de ces 2 articles et seulement dans des massifs forestiers gérés par un personnel nombreux et compétent comme c'est fort heureusement le cas en Guadeloupe.

Reçu le 31 mai 1985. Accepté le 9 novembre 1985.

\section{Remerciements}

Nous tenons à remercier tout particulièrcment la Direction Régionale pour la Guadeloupe de l'Office National des Forêts qui nous a procuré l'aide matérielle indispensable à la réalisation de cette étude, le personnel technique de l'Office National des Forêts de Guadcloupe qui n'a pas ménagé ses efforts pendant toute la durée de l'installation du dispositif expérimental, le personnel technique de la Station de Bioclimatologie de l'INRA de Guadeloupe qui nous a apporté son aide précieuse pendant les opérations de dénombrement et de mesure des semis. Que tous se sentent personnellement concernés par la conduite à son terme de cette expérimentation, vers 1990-1995, est notrc souhait le plus cher. 


\section{Summary \\ Investigations on controlled natural regeneration in the tropical rain forest of Guadeloupe \\ II - Installation and growth of the seedlings after seed felling}

A study on stimulated and controlled natural regeneration was initiated in December 1979 at 4 different sites in the tropical rain forest of Guadeloupe from north to south. At each site, the experimental design consists of three treatments : heavy fellings having removed 28 p. 100 of the trees and 20 p. 100 of the basal area ( 2 plots), light fellings having removed 12 p. 100 of the trees and 5 p. 100 of the basal area ( 2 plots), and a control plot with natural forest.

The present paper reports the results from data collected during the three years after seed felling. The effects of three controlled factors : the site, the species (Dacryodes excelsa, Amanoa caribaea, Simaruba amara and Richeria grandis) and the seed felling intensity on seedling density, their variation with time, regeneration «power» for each species (seedling number per potential seed tree) and seedling growth were evaluated.

Seedling density was significantly different at each site (between 21000 and 64000 per hectare) and for each species (14000 per hectare for Dacryodes excelsa, 22000 for Amanoa caribaea, 1800 for Simaruba amara and 1250 for Richeria grandis). The type of felling had no significant effect on seedling density because of variable number of seed trees from plot to plot, but the regeneration «power» for all species was significantly higher for heavy and light fellings than for the control plot.

Scedling height growth was assessed during approximately one year at two sites. Significant differences were observed between sites and also between species. Annual total height increment was $15.1 \mathrm{~cm}$ for Simaruba amara, $10.9 \mathrm{~cm}$ for Amanoa caribaea and $4.8 \mathrm{~cm}$ for Dacryodes excelsa. Intensity of seed felling had a positive effect on annual height increment for all species : $17.7 \mathrm{~cm}$ with the heavy felling, $6.3 \mathrm{~cm}$ with the light felling and $3.3 \mathrm{~cm}$ with the control plot. Height increment of each species was positively correlated with solar radiation intensity measured under the canopy and also with the inverse of total basal area.

In conclusion, information on ecological requirements of the species under test are given; they may be ranked according to increasing light needs as below : Dacryodes excelsa, Amanoa caribaea, Richeria grandis and Simaruba amara. This behaviour explains the spatial distribution and the frequency of the different species, generally observed in natural forest. Finally, assumptions on silvicultural treatments are proposed.

\section{Références bibliographiques}

Ashton P.S., 1978. The natural forest : Plant biology-Regeneration and tree growth 180-215. In : Natural resources research XIV. Tropical forest ecosystems, U.N.E.S.C.O., Paris.

Barnard R.C., 1950. Linear regeneration sampling. Malayan forester, 13 (3), 129-142.

Catinot R., 1974. Le présent et l'avenir des forêts tropicales humides. Bois et Forêts des Tropiques, 154, 3-26.

DuCREY M., 1975. Utilisation des photographies hémisphériques pour le calcul de la perméabilité des couverts forestiers au rayonnement solaire. Ann. Sci. For, 32 (2), 73-92; 32 (4), 205-221.

Ducrey M., LabbÉ P., 1985. Etude de la régénération naturelle contrôlée en forêt tropicale humide de Guadeloupe. I. Revue bibliographique, milieu naturel et élaboration d'un protocolc expćrimental. Ann. Sci. For., 42 (3), 297-322.

Marshall R.C., 1939. Sylviculture of the trees of Trinidad and Tobago. Oxford University Press, 247 p. 
Office National des Forêts, 1979. Aménagement de la Forêt soumise de Guadeloupe.

Oldeman R.A.A., 1974. L'architecture de la forêt guyanaise. Mémoire ORSTOM $\mathrm{n}^{0}$ 73, ORSTOM éd., Paris, 204 p.

RoLlet B., 1969. Etudes quantitatives d'une forêt dense humide sempervirente de plaine de la Guyane vénézuélienne. Thèse doctorat, Faculté des Sciences, Toulouse, 473 p.

RoLlet B., 1983. La régénération naturelle dans les trouées : un processus général de la dynamique des Forêts tropicales humides. Bois et Forêts des Tropiques, 201, 33-34; 202, 19-24.

Rosevear D.R., Lancaster P.C., 1953. Historique et aspect actuel de la sylviculture en Nigeria. Bois et Forêts des Tropiques, 28, 3-12.

Schulz J.P., 1967. La regeneración natural de la selva mesofitica tropical de Surinam después de su aprovechamiento. Instituto forestal latino-americano de investigación. MeridaVenezuela. Boletin $n^{\circ} 23,3-27$.

WhitMORE T.C., 1978. Gaps in the forest canopy (591-615). In : Tropical trees as living systems. Tomlinson and Zimmerman (eds) London, Cambridge University Press. 